\title{
Influence of age on measured anatomical and physiological interpupillary distance (far and near), and near heterophoria, in Arab males
}

This article was published in the following Dove Press journal:

Clinical Ophthalmology

15 April 2013

Number of times this article has been viewed

\section{Saud A AlAnazi \\ Mana A AlAnazi \\ Uchechukwu L Osuagwu \\ Corneal Research Chair, Department of Optometry and Vision Science, College of Applied Medical Sciences, King Saud University, Kingdom of Saudi Arabia}

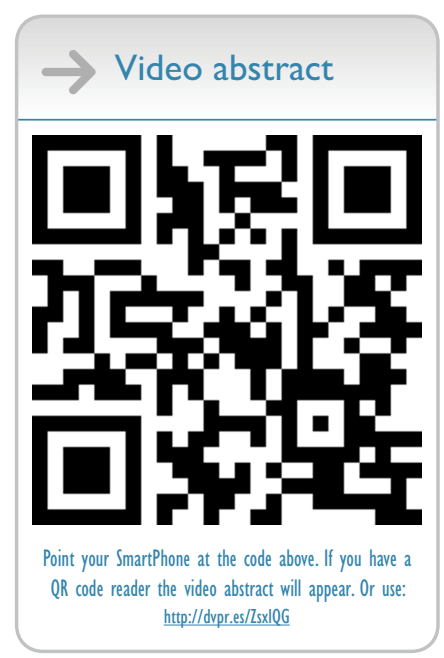

Correspondence: Uchechukwu L Osuagwu Department of Optometry and Vision Sciences, College of Applied Medical Sciences, King Saud University, PO Box 10219, Riyadh II433, Kingdom of Saudi Arabia Tel +966 | 4693530

Fax+966 | 4693535

Email uosuagwu@ksu.edu.sa
Purpose: To compare the effects of age and near phoria on interpupillary distance measured at far (FIPD) and near (NIPD) using the Viktorin's and pupillometer (PD-5) methods.

Methods: Interpupillary distance (IPD) by Viktorin's method and the corneal reflex pupillometer (PD-5) method, as well as near heterophoria by the Saladin near point card, were each obtained on 133 randomly selected normal subjects aged 20-67 years. Comparison within and between techniques, influence of age on IPD, and near heterophoria were assessed.

Results: The mean FIPD varied significantly from the NIPD ( $P<0.001$ for both methods). Overall, FIPD ranged from 56-73 mm, and NIPD ranged from 50-70 mm. For FIPDs of $55 \mathrm{~mm}$, $63 \mathrm{~mm}$, and $71 \mathrm{~mm}$, the corresponding NIPDs were less by $3.9 \mathrm{~mm}, 4.4 \mathrm{~mm}$, and $5.0 \mathrm{~mm}$, respectively, which were measured by Viktorin's method, and $4.0 \mathrm{~mm}, 4.6 \mathrm{~mm}$, and $5.2 \mathrm{~mm}$, respectively, measured by the PD-5 method. Between methods, the limits of agreement were: $-3.9 \mathrm{~mm}$ and $3.2 \mathrm{~mm}(P>0.05)$ for FIPD, and $-3.1 \mathrm{~mm}$ and $2.9 \mathrm{~mm}(P>0.05)$ for NIPD. Both IPDs varied significantly across age groups $(P<0.0001)$. Post hoc analysis revealed a significant variation $(P<0.01$ in both techniques) only in the comparison between age groups 16-25 years and $41-67$ years. The mean difference was $-2.2 \mathrm{~mm}(-4.0 \mathrm{~mm}$ to $-0.3 \mathrm{~mm})$ and $-2.8 \mathrm{~mm}$ $(-4.7 \mathrm{~mm}$ to $-1.0 \mathrm{~mm}$ ) for Viktorin's method for FIPD and NIPD, respectively. For PD-5, the corresponding values were $-1.3 \mathrm{~mm}(-3.2 \mathrm{~mm}$ to $0.4 \mathrm{~mm})$ and $-1.7 \mathrm{~mm}(-4.1$ to $-0.5 \mathrm{~mm})$. Also, the near phoria differed significantly $(P<0.0001)$ across age groups and correlated positively with age $\left(\mathrm{r}^{2}=0.27, P<0.0001\right)$ and NIPD $\left(\mathrm{r}^{2}>0.04 ; P<0.03\right.$, both techniques).

Conclusion: The difference between FIPD and NIPD (about $4.4 \mathrm{~mm}$ in Arab males) was observed independent of the technique used. The Viktorin's and the PD-5 methods of IPD assessment resulted in similar values, and therefore, could be interchangeably used. However, caution is advised in cases of high power refractive corrections as the difference could vary from $-4 \mathrm{~mm}$ to $+3 \mathrm{~mm}$ (FIPD) and $-3 \mathrm{~mm}$ to $+3 \mathrm{~mm}$ (NIPD). Both IPDs have demonstrated an increase until the patients are in their early 40s, and a slight decrease has been observed thereafter. Age and NIPD were significantly associated with heterophoria in our subjects.

Keywords: phoria, interpupillary distance, Viktorin's method, pupillometer, Saladin near point card, age

\section{Introduction}

Interpupillary distance (IPD) is the distance between the centers of the pupils. It determines the stereo separation of the two images, which are combined in the brain to produce stereo perception. It has been shown to correlate with head size, and as such is an important parameter used by the optical industries in the design and manufacture of lenses. ${ }^{1}$ Knowledge of mean IPD is important in the satisfactory performance of finished spectacles. Ophthalmic lenses are positioned before the eyes such that their 
optical centers coincide with the centers of the pupils in order to eliminate unwanted prismatic effects. ${ }^{2}$ Normative values of IPD are also useful in surgery after facial trauma, and have been applied in the selection of artificial teeth. ${ }^{3}$ Juberg et $\mathrm{al}^{4}$ listed a number of syndromes in which the knowledge of IPD could be helpful in their diagnosis (eg, ocular hypertelorism, Waardenburg syndrome, mongolism, and so on). More recently, it has been applied in the diagnosis of fetal alcohol syndrome. ${ }^{5}$

Several studies have demonstrated an increase in far IPD (FIPD) with age in both males and females. ${ }^{6-12}$ A parallel and steady increase was evident for both genders from infancy through to puberty, ${ }^{9}$ and this increase slows down from the time individuals reach their middle 20 s to their late 30 s. ${ }^{13}$ Afterwards, a slight decrease occurs in older subjects. ${ }^{7,9}$ Fesharaki et $\mathrm{al}^{13}$ showed that mean IPD in an Iranian population increased $4.8 \mathrm{~mm}$ during the second decade, $1.7 \mathrm{~mm}$ during the third decade, and $0.6 \mathrm{~mm}$ during the fourth and fifth decades of life. Another study showed an increase in FIPD and NIPD of female Arabs across age groups ( 7 years to 40 years). ${ }^{6}$ Interestingly, Chen and O'Leary ${ }^{8}$ also showed that IPD changes significantly with age, but that the oculomotor control system for convergence can compensate for these age-related changes in IPD during childhood.

Vernier calipers, video centration devices, ${ }^{14}$ photographs, ${ }^{15,16}$ the ruler-Viktorin's method, ${ }^{6,9}$ and pupillometers, ${ }^{14,17,18}$ have all been used in the determination of human IPDs. Essentially, these techniques operate on one of two principles: measuring the distance between the two principal corneal reflexes (physiological IPD), or measuring the distance between common points on each eye (anatomical IPD). ${ }^{18}$ The Viktorin's method and pupillometer have been commonly employed in the clinical determination of IPD. While the former measures IPD by determining the distances between appropriate features in each eye (in this case, the temporal and nasal pupil margins in each eye respectively), the latter is able to measure IPD using the corneal reflex, and has been shown to give more repeatable results at far and near IPDs. ${ }^{2,17-19}$ The anatomical IPD (determined by the Viktorin's method) locates the line of sight or optical axis of the eye, while the physiological IPD (determined by pupillometer) locates the visual axis. Since these two axes are not coincident, ${ }^{18}$ the anatomical IPD is usually $0.5-1.0 \mathrm{~mm}$ larger than the physiological IPD. ${ }^{17}$

A search of the published literature revealed that very few studies have evaluated the differences in anatomical and physiological IPD as measured by the Viktorin's method and pupillometer, respectively. Though the results of these studies have been contradictory, they have also either presented data on a few subjects, ${ }^{2,18,20,21}$ or have considered only the FIPD while neglecting the NIPD, ${ }^{17,19}$ which is important during the selection and manufacture of reading glasses, as shown in previous studies. ${ }^{22-24}$ Elliot and Green's ${ }^{23}$ study found a high prevalence of induced horizontal $(60 \%)$ and vertical prism $(32 \%)$ beyond the tolerance levels stipulated in the International Organization for Standardization ISO 16034:2002 after examining 322 near-vision ready-made spectacles and strongly recommended that manufacturers use a centration distance for near-vision ready-made spectacles that is similar to an average near (and not distance) IPD. Pointer ${ }^{24}$ also observed that in Caucasians, the NIPD stabilizes across the presbyopic age span (41-80 years), and as such advised the need to specify two lens centration distances in order to cater to the gender of the intended Caucasian wearer: namely $62 \mathrm{~mm}$ for male and $59 \mathrm{~mm}$ for female presbyopes.

Comparing different methods of IPD measurement, Holland and Siderov ${ }^{17}$ reported that FIPD measured using the Viktorin's method did not differ significantly from that obtained by pupillometer on the first measurement, whereas on retest, the former returned an FIPD that was statistically significantly greater than the latter by about $0.40 \mathrm{~mm}$. However, the authors attributed this small difference to a statistical quirk. Another comparative study conducted more than a decade ago reported that both techniques measured FIPDs that were clinically similar, and as such, could be used interchangeably in practice. ${ }^{18}$ In view of the controversy that exists on the differences in FIPD obtained using the Viktorin's method and pupillometer; the nonavailability of data comparing the difference between FIPD and NIPD obtained by both devices; and the potential significance of the results in our population, we designed this study using both techniques. The aim of this study was: (1) to compare the differences in far and near anatomical and physiological IPD as measured by the Viktorin's and pupillometer methods, respectively, so as to be able to predict the FIPD when the NIPD can be measured (for instance, in a case where the examiner is amblyopic in one eye); (2) to assess and compare the limits of agreement (LoA) between anatomical and physiological IPD at both distances as obtained by both devices; and (3) to evaluate the changes in anatomical and physiological FIPD and NIPD across various ages. In addition, we also assessed the influence of age and NIPD on near heterophoria.

\section{Methods}

\section{Subjects}

A total of 133 Saudi males with a mean age of $30.4 \pm 13.0$ years (mean \pm standard deviation; range: 20 to 67 years) were 
randomly recruited from a population of students from the College of Applied Medical Sciences, and relatives of subjects visiting the optometry clinic for routine eye examinations between December 2012 and January 2013 for participation in this study. We choose the lower band of the age limit in order to minimize the reported effects of menstruation changes that occur during childhood physical growth changes, ${ }^{6}$ while the upper band of the age limit was chosen to minimize confounding factors arising from the increased laxity of the soft tissues around the orbit of the elderly subjects. ${ }^{9}$ However, the decision to include only males was made because data was collected from the College of Applied Medical Sciences Optometry department male-only clinic, and/or to enhance the direct comparison of our findings with those of Osuobeni and al-Gharni ${ }^{15}$ which was carried out a decade ago on male samples of a similar population.

Subjects were included if there was no ocular pathology, if they recorded an acuity of $6 / 6$ or better, and had no binocular vision anomalies. Distance refractive correction $\geq \pm 6.00$ diopters sphere and $\geq-2.00$ diopters cylinder, and anisometropia of $\geq 2.00$ diopters spherical equivalent, excluded the subjects' data from inclusion in the sample.

Ethical approval was obtained from the College of Applied Medical Sciences Research Ethics Committee. The purpose of the study was explained, and each subject signed an informed consent sheet. The study adhered to the principles of the 1967 Helsinki declaration (as modified in Edinburgh, UK in 2000).

\section{Data acquisition}

IPD was obtained on each subject by the same examiner using two methods: Viktorin's method and a commercial corneal reflection digital pupillometer, PD-5 (Topcon Corporation, Tokyo, Japan), which has been in everyday use in the clinic. This was followed by determination of near heterophoria using the Saladin Near Point Balance Card version 1.0 (Saladin card; Bernell Corp, Mishawaka, IN, USA). No recalibration of the pupillometer was done specifically for this study. Determination of the IPD measurement methods to be first used was done by randomization via a series of random numbers generated from the Microsoft Word, 2010 (Microsoft Corporation, Redmond, WA, USA). One examiner (SA) obtained all IPD measurements, while a second examiner (UO) who recorded the pupillometer readings (so the first examiner [SA] had no knowledge of the pupillometer readings), obtained measurements of near heterophoria in all subjects.

\section{Methods used to measure IPD and heterophoria \\ Viktorin's method}

This method was adopted as it has remained the most widely taught and practiced IPD measurement technique, ${ }^{9}$ and since it was the most widely cited in published data involving the assessment of IPD. ${ }^{7}$ The FIPD and NIPD were simultaneously measured by one examiner using a millimeter rule, as outlined by Sasieni. ${ }^{25}$ The subject was seated opposite to, on the same level as, and within arm's length of the examiner in a well-lit optometry consulting room. A PD rule calibrated in millimeters was laid across the subject's nose and lightly supported by the examiner's left hand. The subject was instructed to keep both eyes open and to fixate at the examiner's open left eye; using this eye, the examiner aligned the "zero" mark on the PD rule with the edge of the temporal limbus of the subject's right eye, and sighted the point on the ruler that corresponded to the nasal limbus of the subject's left eye. This measurement is equivalent to the NIPD value. Keeping the ruler still, the examiner then closed his left eye and opened his right eye, while instructing the subject to move fixation across to the examiner's open right eye. The examiner also sighted the point on the ruler that corresponded to the nasal limbus of the subject's left eye. This measurement is equivalent to the FIPD. The same procedure was repeated from the right to left, and the averages were recorded.

\section{Pupillometer method}

The examiner aligned the crosshairs of the pupillometer, which was set for a $40 \mathrm{~cm}$ distance with the corneal reflexes of the subject. This is equivalent to NIPD. The pupillometer was then reset at an infinite distance and realignment of the crosshairs with the subjects' corneal reflexes was again performed. This is equivalent to FIPD.

\section{Heterophoria measurement}

A horizontal dissociated heterophoria test was performed for every participant for near heterophoria; however, vertical heterophoria was not assessed. It is acknowledged that convergence/divergence is an active mechanism, and accommodative vergence refers to a blur-driven change in the horizontal alignment of the two eyes. ${ }^{26}$ In 2001, the Saladin card was designed by Saladin to provide a quick measurement device for several visual function parameters at near: near VA (logarithm of the minimum angle resolution), horizontal and vertical dissociated heterophoria, horizontal and vertical associated heterophoria, horizontal and 
vertical fixation disparity, accommodative facility, vergence facility, and monocular estimation method retinoscopy for accommodation lag. The Saladin Near Point Balance Card was adopted for use in this study not only because of its versatility, but also because it is considered to be a faster procedure than the Sheedy disparometer, ${ }^{27}$ and has a black disk with words surrounding the black discs, possibly acting to control and/or stabilize accommodation during the test; therefore, the print supposedly forms an additional peripheral lock. It has also been found to be the most repeatable subjective dissociated phoria test. ${ }^{28,29}$ The Saladin card has the advantage of being an inexpensive, small, lightweight, and portable facility, ${ }^{28}$ making it particularly useful in nontraditional optometric settings such as in schools and nursing homes. ${ }^{30,31}$

Near heterophoria was recorded using a Maddox rod and Saladin Card placed at eye level at $40 \mathrm{~cm}$ with an illumination level of 300 lux measured using a Minolta illuminance meter (Konica Minolta Sensing Singapore Pte Ltd, Shanghai, People's Republic of China). A light-emitting diode light source was used to produce a spotlight source behind the Saladin card, which was seen through the central pinhole located at the intersection of the horizontal and vertical scales. The subject was instructed to report the position of the vertical red line in relation to the central spot light (left or right), and the number intersected on the scale (measured in prism diopters [pd]), which represented the dissociated heterophoria. Where the subjects reported heterophoria between two line scales, the mid-value was recorded.

\section{Data analysis}

All visual data and background information for each subject was entered into a computer database, but only those relevant to the study were exported for each subject for analysis into a Microsoft Excel (Microsoft Corporation) spreadsheet. GraphPad InStat version 3.00 (GraphPad Software Inc, San Diego, CA, USA) statistical software was used for subsequent standardized analysis. To achieve a statistical power of $85 \%$ at a significance level of $\alpha=0.05$, 123 subjects were required for this study. This sample size calculation was determined from an initial sample of 15 subjects using the statistical freeware $\mathrm{G}^{*}$ Power (version 3; Heinrich Heine Universität Düsseldorf, Dusseldorf, Germany).

Mean values of age in years, FIPD and NIPD in millimeters, and heterophoria in pd were all descriptively analyzed. Comparisons of FIPD and NIPD within technique and between techniques in all subjects were performed using repeated measures analysis of variance (ANOVA). The columns analyzed were columns of FIPD versus NIPD (Viktorin's method) versus FIPD versus NIPD (PD-5 method).

We assessed the agreement at both distances within and between techniques by obtaining the difference between IPD measured at both distances by the same technique (withintechnique) and between techniques, and determined the $95 \%$ LoA (mean difference $\pm 1.96 \times$ standard deviation of the differences). The LoA defined the region of IPD measurements within which there was a $95 \%$ probability that the differences occurred due to measurement variability and a $5 \%$ probability that the difference was real and not due to measurement error. ${ }^{32}$

To assess the reported differences between age groups in relation to IPD and phoria, ${ }^{8,9,11,16,33-35}$ subjects were divided into three age groups and further analysis was conducted between age groups using one-way ANOVA. The age groups were: $16-25$ years $(\mathrm{n}=90) ; 26-40$ years $(n=10)$ and $40-67$ years $(n=33)$. The association of age with heterophoria and IPD with heterophoria were both tested and analyzed across individuals using a linear regression model to assess the rate of change in each association, as well as the significance level of any existing relationship.

\section{Results}

\section{Average values of all measurements and subjects}

Descriptive statistics for the mean values (in millimeters) of the two techniques used in the measurement of IPD are given in Table 1. In general, the mean FIPD and NIPD in Saudi males ranged between $56-73 \mathrm{~mm}$ and $50-70 \mathrm{~mm}$, respectively, in spite of the technique used.

\section{Limits of agreement in IPD obtained by same technique (within-technique) and across techniques (between techniques)}

A repeated measures ANOVA conducted using four levels of the factor method of measurement (essentially two corresponding to each technique - one for near and one for far) revealed a significant difference in IPD across the methods of measurement $\left(\mathrm{F}_{3,612.5}=27.4, P<0.0001\right)$. Bonferroni corrected posttest analysis showed that the measurement of IPD was not dependent on the method of measurement employed $(P>0.05)$; rather, it was dependent on the distance at which IPD measurement was obtained $(P<0.001)$. On the average, the FIPD obtained by the Viktorin's method and 
Table I Mean values \pm SD, range of anatomical and physiological IPDs as measured by the Viktorin's and Topcon PD-5 pupillometer method, respectively, for FIPD and NIPD in millimeters

\begin{tabular}{lllllll}
\hline Method & $\mathbf{n}$ & Mean FIPD \pm SD & Range & Mean NIPD \pm SD & Range & Paired test \\
\hline Viktorin's & 133 & $62.5 \pm 3.2$ & $56-72$ & $58.1 \pm 3.4$ & $50-70$ & $0.00 I^{*}$ \\
PD-5 & 133 & $62.8 \pm 3.2$ & $56-73$ & $58.2 \pm 3.4$ & $>0.05 * *$ & $0.00 I^{*}$ \\
P-value & & $>0.05 * *$ & & &
\end{tabular}

Notes: Results of statistical analyses were performed on 133 normal subjects aged 20-67 years. P-values are the results of repeated measures ANOVA. *FIPD versus NIPD obtained by the same technique (within-technique); **Viktorin's method-measured IPD versus PD-5 pupillometer measured IPD (between-technique) for FIPD and NIPD. Abbreviations: SD, standard deviation; IPDs, interpupillary distances; FIPD, far interpupillary distance; NIPD, near interpupillary distance; ANOVA, analysis of variance.

the PD-5 methods were, respectively, $4.4 \mathrm{~mm}$ and $4.6 \mathrm{~mm}$ greater than the NIPD obtained by the same technique. The magnitude of the $95 \%$ LoA between FIPD and NIPD is shown in the Bland-Altman plot (Figure 1 [Viktorin's method]; Figure 2 [PD-5 method]). Linear regression analysis plot of the differences in FIPD and NIPD as a function of FIPD obtained by each technique (Figure 3) are best explained by the following equations:

Viktorin's method: y $($ FIPD - NIPD $)=0.07 \times$ FIPD $(\mathrm{mm})$

PD-5 method: y $($ FIPD - NIPD $)=0.0731 \times$ FIPD $(\mathrm{mm})$.
Between techniques, the width of the $95 \%$ LoA was wider for FIPD (7.1 mm; Figure 4) than NIPD (5.9 mm; Figure 5), with the pupillometer consistently measuring IPDs larger than the Viktorin's method. However, the differences were not statistically significant at both far and near $(P>0.05$, for both).

\section{Comparison of interpupillary distance (IPD) measures between age groups}

Subjects were grouped into age groups of 16-25 years, 26-40 years, and 41-67 years, representing young adults, adults, and older adults, respectively. These groupings were selected to enhance the analysis and comparison of the current data

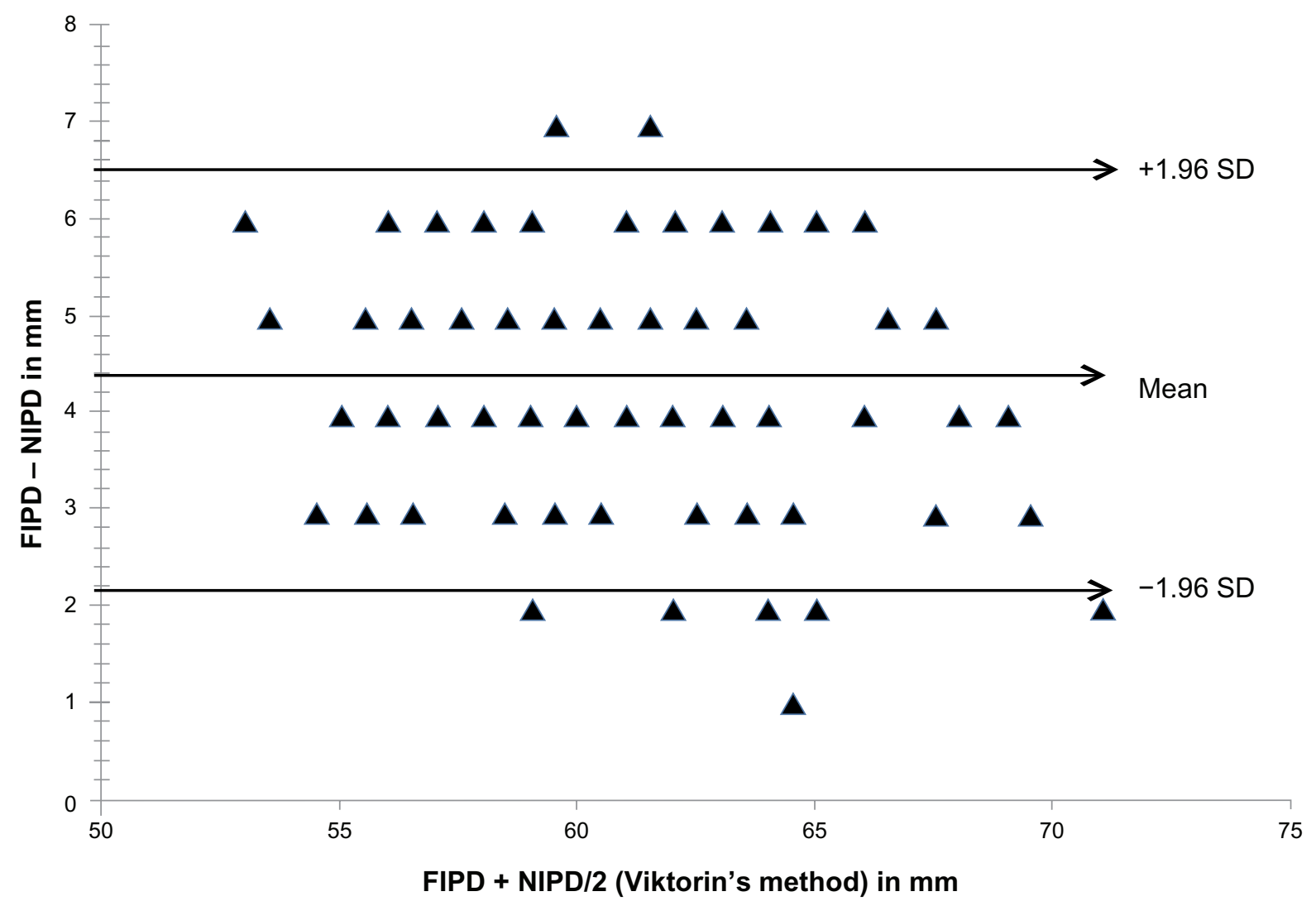

Figure I Limit of agreement (LoA) in anatomical interpupillary distance (IPD) measured for FIPD and NIPD using the Viktorin's method, in millimeters. Note: The FIPD was on the average $4.4 \mathrm{~mm} \pm \mathrm{I} . \mathrm{I} \mathrm{mm}$ significantly higher $(P<0.00 \mathrm{I})$ than the NIPD.

Abbreviations: LoA, limit of agreement; IPD, interpupillary distance; FIPD, far interpupillary distance; NIPD, near interpupillary distance; SD, standard deviation. 


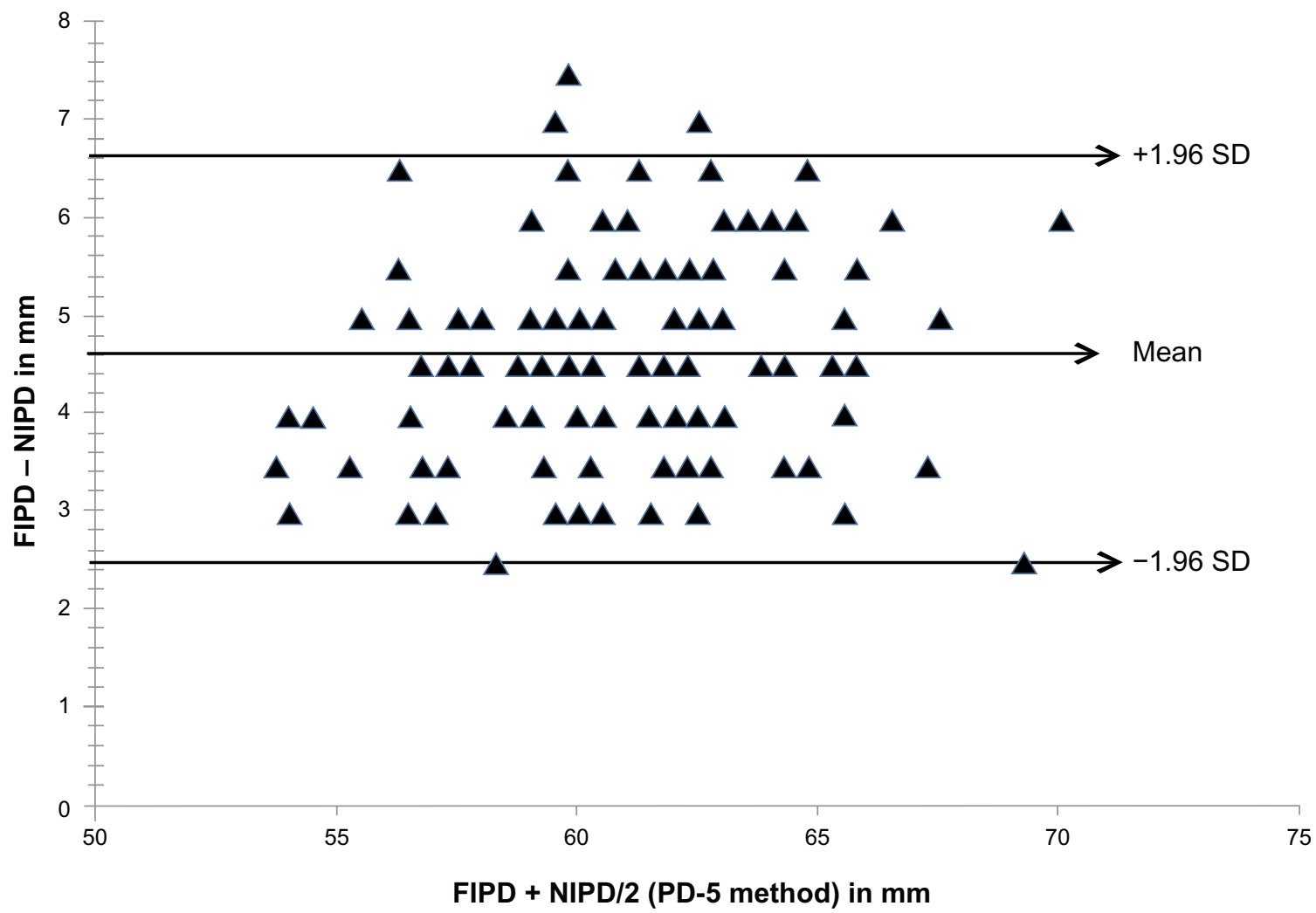

Figure 2 Limit of agreement in physiological interpupillary distance IPD measured for FIPD and NIPD using the pupillometer (PD-5) method, in millimeters. Note: The FIPD was on the average $4.6 \mathrm{~mm} \pm 1.0 \mathrm{~mm}$ significantly higher $(P<0.00 \mathrm{I})$ than the NIPD.

Abbreviations: IPD, interpupillary distance; FIPD, far interpupillary distance; NIPD, near interpupillary distance; SD, standard deviation.

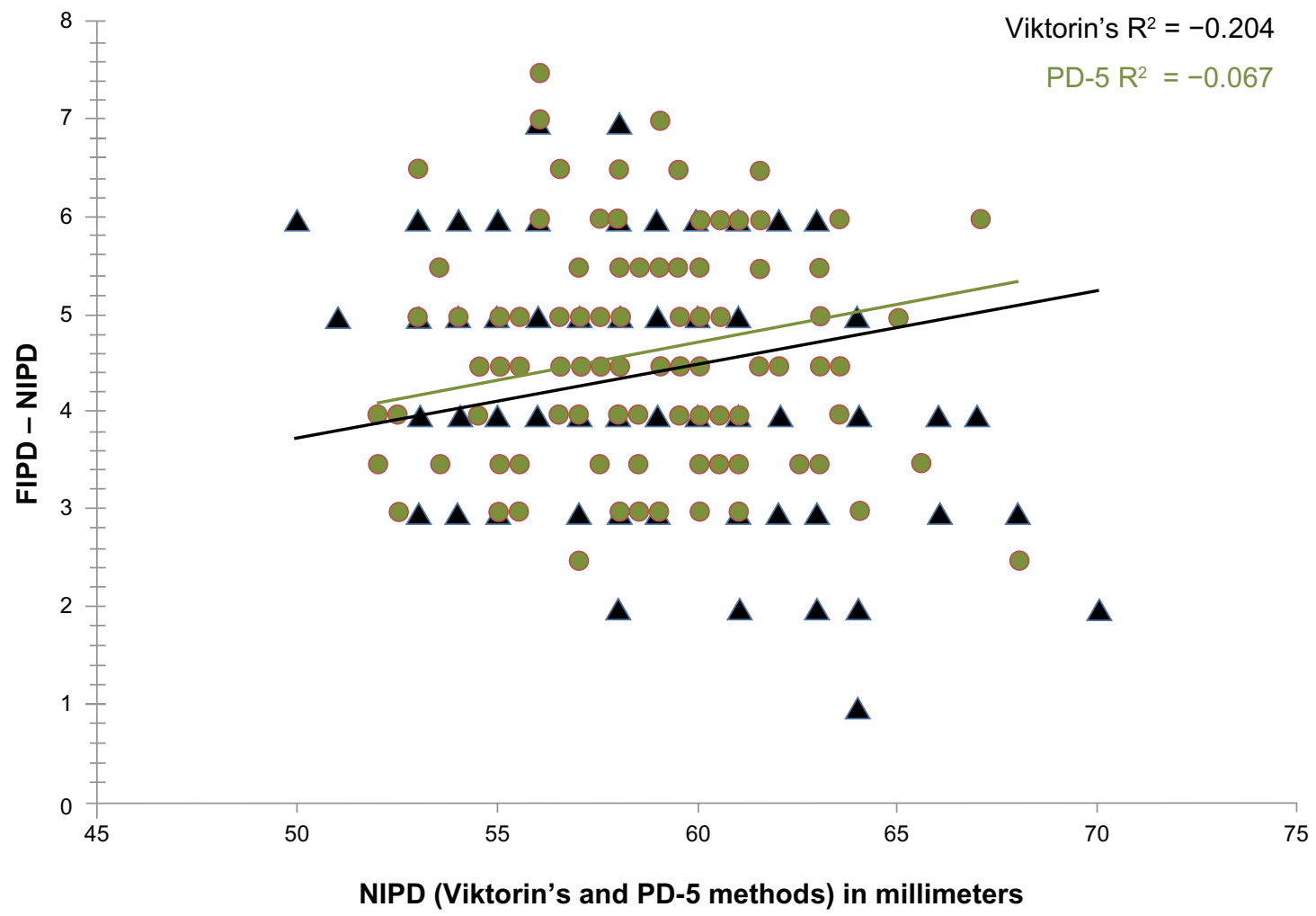

Figure 3 Linear regression plot of the difference in IPD for FIPD and NIPD as a function of NIPD in millimeters. Abbreviations: IPD, interpupillary distance; FIPD, far interpupillary distance; NIPD, near interpupillary distance. 
Dovepress

Influence of age on IPD and near phoria

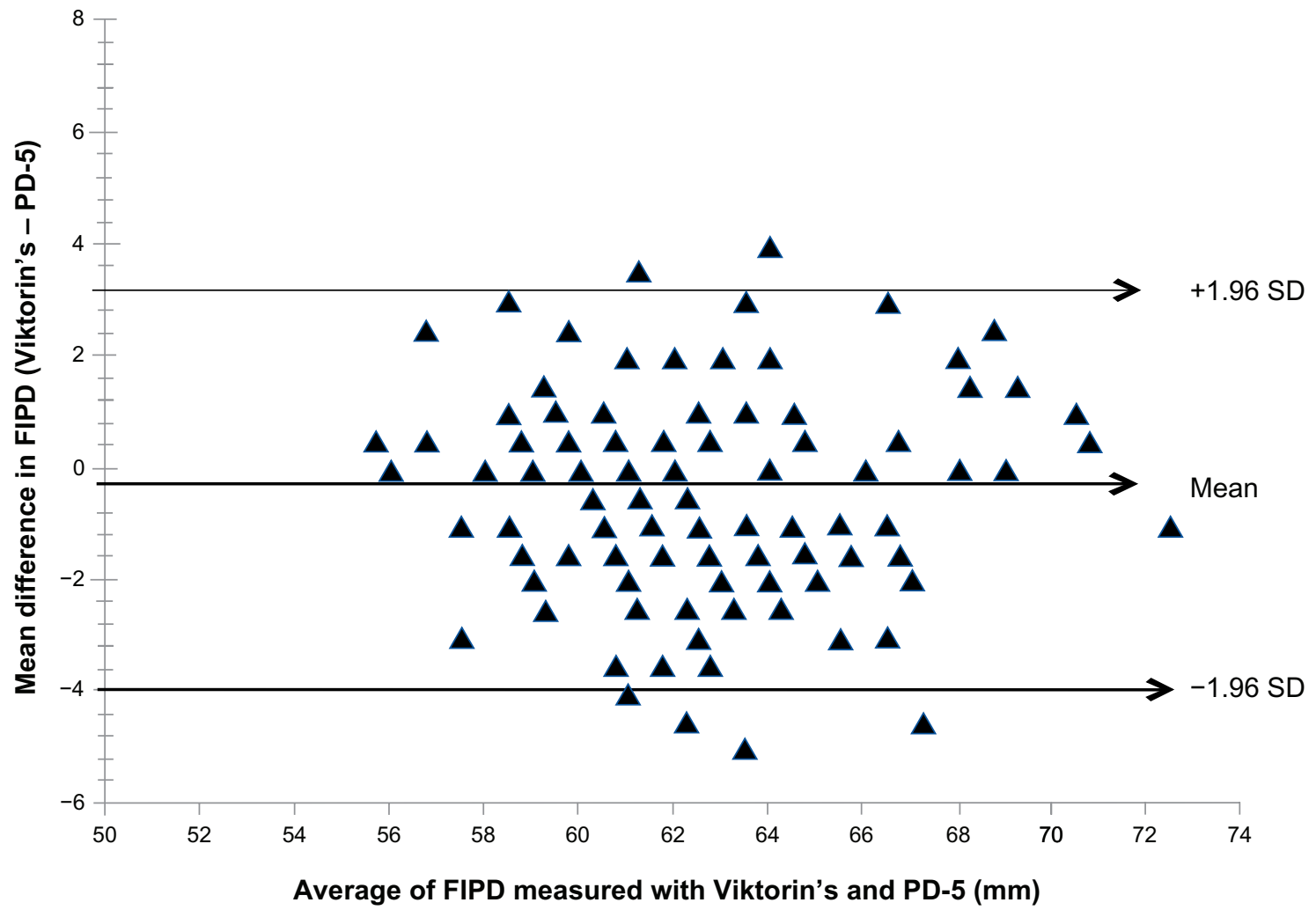

Figure 4 Limit of agreement between anatomical and physiological IPD as measured by Viktorin's and PD-5 pupillometer methods, respectively, at FIPD, in millimeters. Note: The difference between FIPD obtained by both techniques was not statistically significant $(P>0.05)$.

Abbreviations: IPD, interpupillary distance; FIPD, far interpupillary distance; SD, standard deviation.

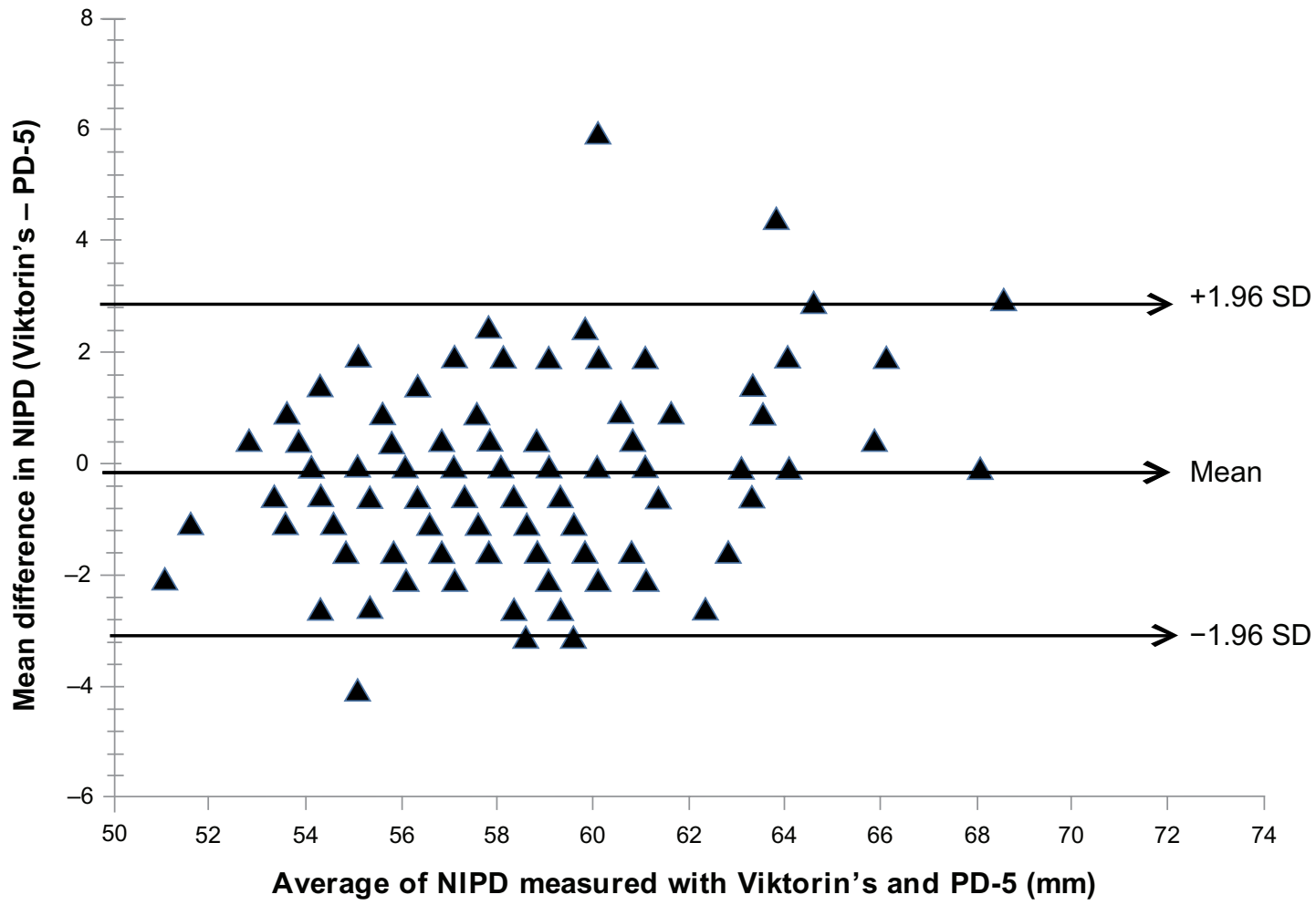

Figure 5 Limit of agreement between anatomical and physiological IPD as measured by Viktorin's and PD-5 pupillometer methods respectively, for NIPD, in millimeters. Note: The difference between NIPD obtained by both techniques was not statistically significant $(P>0.05)$.

Abbreviations: IPD, interpupillary distance; NIPD, near interpupillary distance; SD, standard deviation.

Clinical Ophthalmology 2013:7

submit your manuscript | www.dovepress.com

717

Dovepress 
with those of other published data on both similar and different populations, ${ }^{6,7,9,10,13}$ using similar age classification systems.

Figure 6 shows the mean values of FIPD, NIPD in mm, and heterophoria in prism diopters (pd) across the different age groups. The picture emerging from the results of the one-way ANOVA of the age grouped data is shown in Table 2. Using the Viktorin's method, FIPD differed significantly from NIPD across all age groups $\left(\mathrm{F}_{5,263}=33.9, P=0.0001\right)$. Post hoc analysis showed statistically significant differences between age groups 16-25 years and 41-67 years with IPD values measured at far $(P<0.05)$ and near $(P<0.001)$. All other age group comparisons of mean IPDs measured at both distances by the Viktorin's method showed nonstatistically significant differences ( $P>0.05$, for both FIPD and NIPD).

FIPD and NIPD obtained by the PD-5 method also varied significantly $\left(\mathrm{F}_{5,263}=32.8, P=0.0001\right)$ across all age groups. However, post hoc analysis only showed statistically significant differences $(P<0.01)$ in NIPD when the age group 16-25 years was compared to the 41-67 year age group.
The $95 \%$ LoA and results of the ANOVA have been presented in Table 2.

\section{Influence of age and near interpupillary distance (NIPD) on heterophoria}

In the analysis, a negative sign (-) denotes esophoria, a positive sign $(+)$ denotes exophoria, and zero (0) denotes orthophoria. Across all age groups, the difference in the amount of measured heterophoria was statistically significant $\left(\mathrm{F}_{2,131}=21.9, P<0.0001\right)$. On post hoc analysis between age groups, the difference in measured phoria was statistically significantly different between groups (16-25 years and 26-40 years [mean difference: $-2.9, P<0.05$ ], 16-25 years and 41-67 years [mean difference: -4.8 , $P<0.001])$. There was no statistically significant difference in the comparison between the 26-40 year and 41-67 year age groups $(P>0.05)$. The prevalence of near orthophoria, exophoria, and esophoria was 26 participants (19.5\%), 38 participants $(28.6 \%)$, and 69 participants $(51.9 \%)$, respectively.

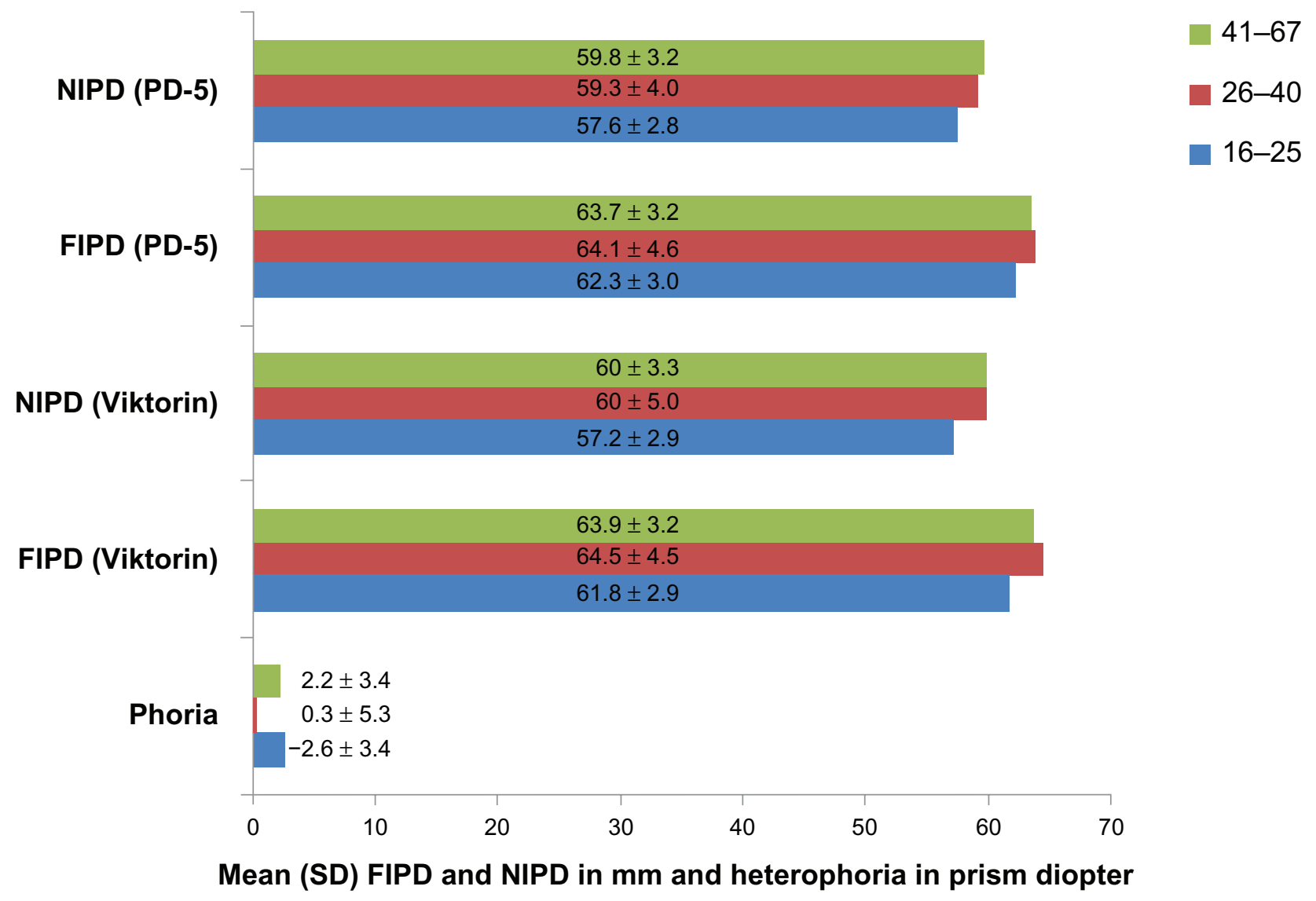

Figure 6 Age-grouped mean values of anatomical and physiological IPDs as measured by the Viktorin's and PD-5 pupillometer methods, respectively, for FIPD and NIPD in millimeters.

Note: Heterophoria in prism diopters, obtained on 133 normal subjects aged 20-67 years.

Abbreviations: IPD, interpupillary distance; FIPD, far interpupillary distance; NIPD, near interpupillary distance; SD, standard deviation. 
Table 2 Results of age-group comparisons of mean anatomical and physiological IPDs for FIPD and NIPD in millimeters, withintechnique (Viktorin's and PD-5 pupillometer methods)

\begin{tabular}{llll}
\hline Group comparison & MD (LoA) FIPD & MD (LoA) NIPD & Unpaired test \\
\hline I6-25 years versus 26-40 years (Viktorin) & $-2.8(-5.2 /+0.3)$ & $-2.8(-5.8 /+0.2)$ & $>0.05^{*},>0.05^{* *}$ \\
I6-25 years versus 4I-67 years (Viktorin) & $-2.2(-4.0 /-0.3)$ & $-2.8(-4.7 /-I .0)$ & $<0.05^{*},<0.00 I^{* *}$ \\
$26-40$ years versus 4I-60 years (Viktorin) & $0.6(-2.1 /+3.8)$ & $-0.0(-3.3 /+3.2)$ & $>0.05^{*},>0.05^{* *}$ \\
I6-25 versus 26-40 years (PD-5) & $-1.8(-4.8 /+I .1)$ & $-1.7(-4.7 /+1.2)$ & $>0.05^{*},>0.05^{* *}$ \\
I6-25 versus 4I-67 years (PD-5) & $-1.3(-3.2 /+0.4)$ & $-2.3(-4.1 /-0.5)$ & $>0.05^{*},<0.0 I^{* *}$ \\
$26-40$ versus 4I-67 years (PD-5) & $0.4(-2.8 /+3.6)$ & $-0.5(-3.7 /+2.7)$ & $>0.05^{*},>0.05^{* *}$ \\
\hline
\end{tabular}

Notes: P-values are the results of one-way ANOVA. *P-values comparing FIPDs; $* *$-values comparing NIPDs. LoA $=95 \%$ LoA (LoA: mean difference \pm I.96 $\times$ SD of the differences).

Abbreviations: IPD, interpupillary distances; FIPD, far interpupillary distance; NIPD, near interpupillary distance; ANOVA, analysis of variance; MD, mean difference; LoA, limits of agreement; SD, standard deviation.

The amount of near heterophoria (in pd) was moderately correlated with the age of subjects $\left(\mathrm{r}^{2}=0.27, P<0.0001\right.$, Figure 7), but was weakly and positively associated with PD-5 measured NIPD (slope: $0.26, \mathrm{r}^{2}=0.037 ; P=0.027$; Figure 8), and the Viktorin's method-measured NIPD (slope: $0.27, \mathrm{r}^{2}=0.0476 ; P=0.0116$; Figure 8). Overall, there was a predominance of exophoria for NIPDs $>63 \mathrm{~mm}$, and esophoria for NIPDs $<62 \mathrm{~mm}$, despite the technique used.

\section{Discussion}

Our results show that the mean anatomical FIPD and NIPD in Saudi males ranged from $56-72 \mathrm{~mm}$ and $50-70 \mathrm{~mm}$, as well as from $56-73 \mathrm{~mm}$ and $52-68 \mathrm{~mm}$ for the physiological FIPD and NIPD, respectively. Dodgson ${ }^{33}$ reported that in a vast majority of adults, FIPD values lie within the range $50 \mathrm{~mm}$ to $75 \mathrm{~mm}$. Osuobeni and al-Musa ${ }^{7}$ reported similar findings for male anatomical FIPD $(58-73 \mathrm{~mm})$ and a slightly wider NIPD (56-70 mm) in Arabs aged 26-55 years. Fesharaki et al ${ }^{13}$ reported the mean IPD in adult Iranian subjects to be $61.1 \mathrm{~mm} \pm 3.5 \mathrm{~mm}$ in women, and $63.6 \mathrm{~mm} \pm 3.9 \mathrm{~mm}$ in men. Hofstetter ${ }^{36}$ also reported that the mean IPD in $90 \%$ of white adults in the United States of America lie between $60 \mathrm{~mm}$ and $70 \mathrm{~mm}$, while the majority (99.8\%) of his subjects' IPDs were between $55 \mathrm{~mm}$ and $75 \mathrm{~mm}$. More recently,

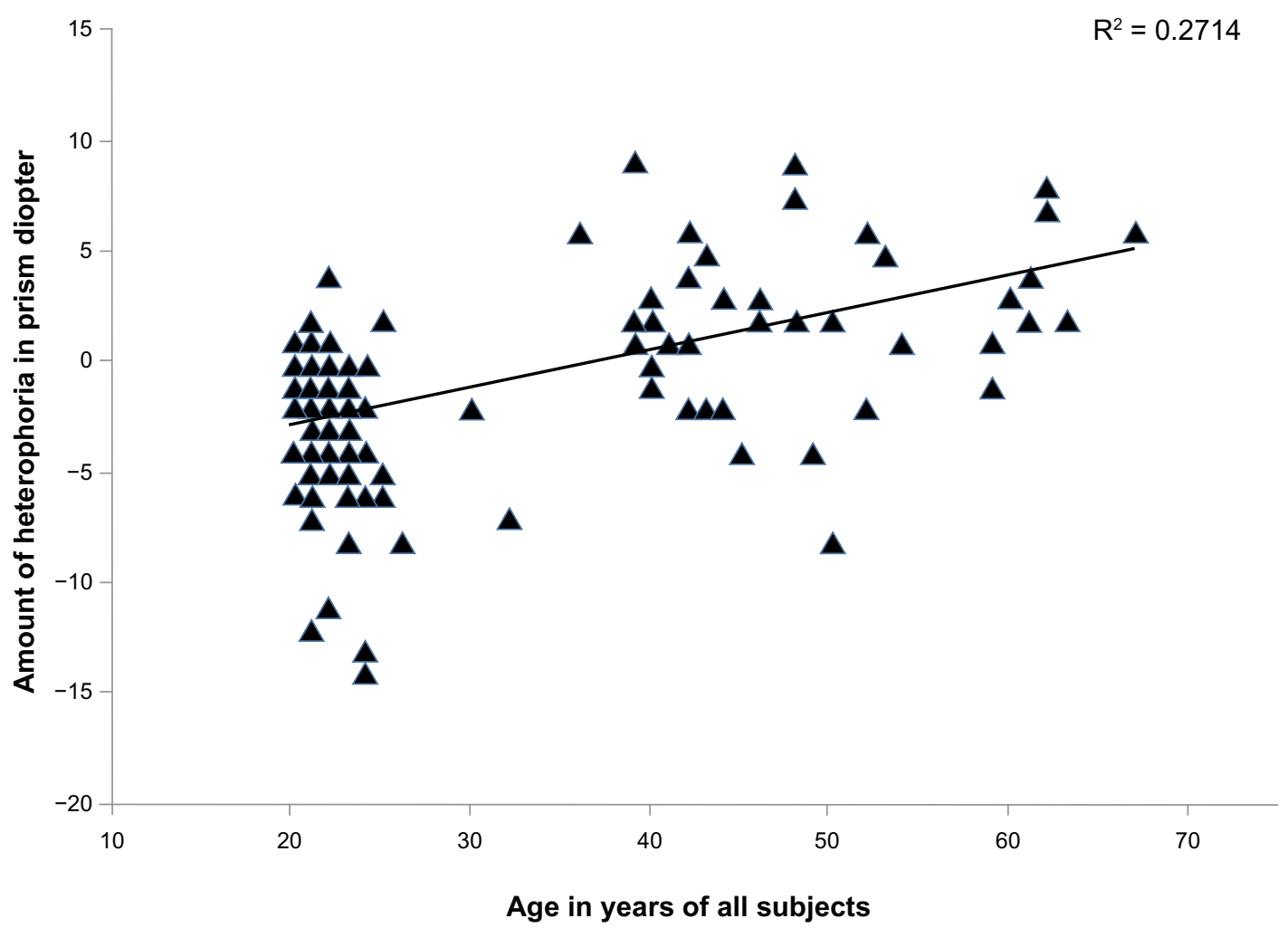

Figure 7 Amount of near heterophoria in prism diopters as a function of the subjects' ages in years. 


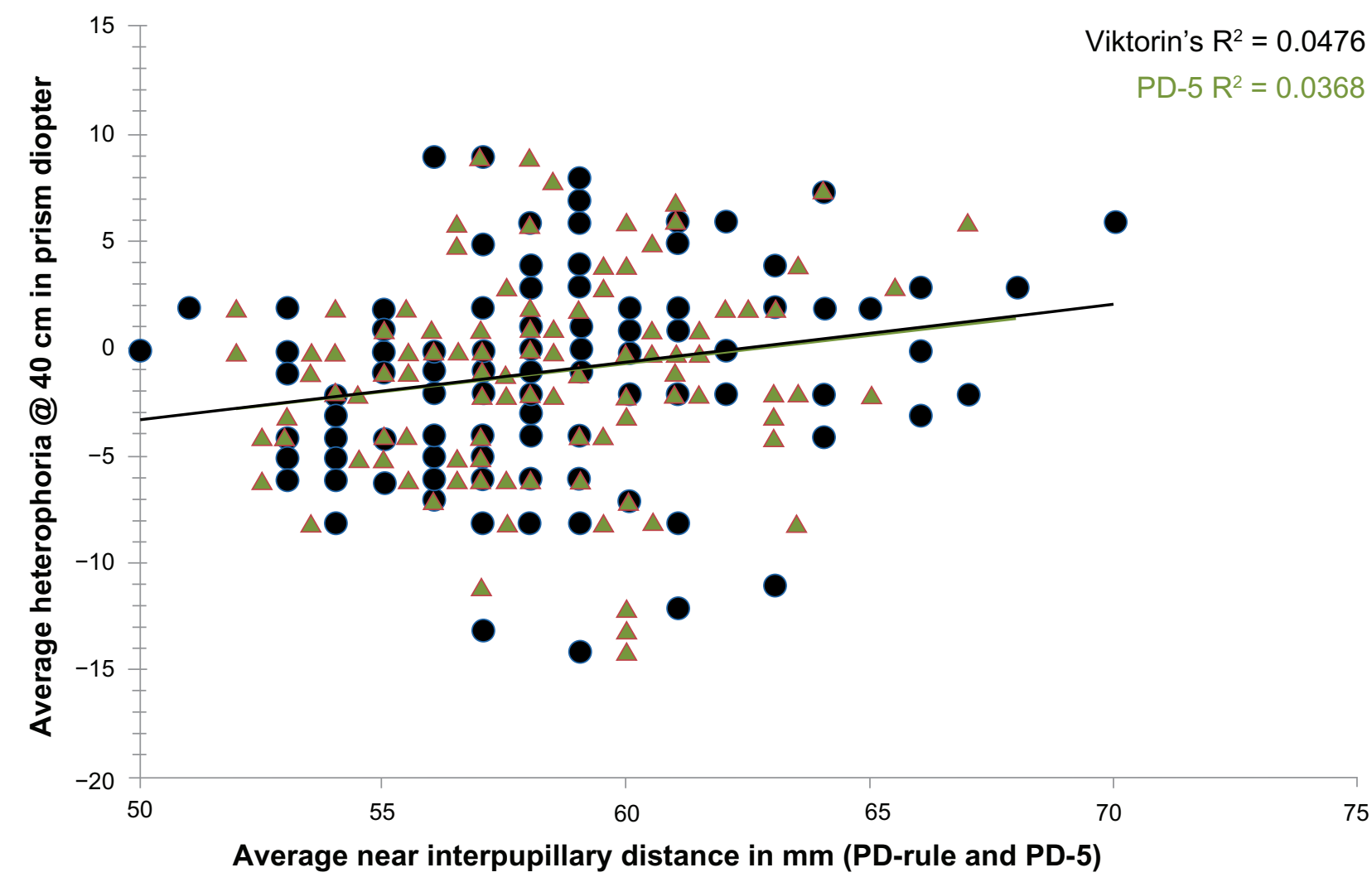

Figure 8 Amount of near heterophoria in prism diopters (pd) as a function of near Anatomical and Physiological Interpupillary distances (IPDs) as measured by the Viktorin's and PD-5 pupillometer method, in millimeters.

Caucasian male and female mean IPDs have been reported to be $65.3 \mathrm{~mm} \pm 2.8 \mathrm{~mm}$ and $62.3 \mathrm{~mm} \pm 2.8 \mathrm{~mm}$ (far), and $62.2 \mathrm{~mm} \pm 2.7 \mathrm{~mm}$ and $59.3 \mathrm{~mm} \pm 2.8 \mathrm{~mm}$ (near), respectively. ${ }^{24}$ The values reported in the current study fall within the range reported by the cited studies. ${ }^{7,24,33,36}$ Compared to the study involving Arabs, ${ }^{7}$ the difference in the measured anatomical NIPD reported in the current study could be attributed to the inclusion of much older subjects (up to 67 years). NIPD decreased slightly (although the difference was not statistically significant) between the 26-40 and 41-67 year age groups in the current study, which could have resulted in the smaller NIPD reported. A similar decrease was also shown in a more recent study where NIPD was shown to decrease from $62.2 \mathrm{~mm} \pm 2.7 \mathrm{~mm}$ (at the age of $57 \pm 9.6$ years) to $59.3 \mathrm{~mm} \pm 2.8 \mathrm{~mm}$ (at the age of $55.9 \pm 9.7$ years) in Caucasian presbyopic males. ${ }^{24}$

The collation of NIPD data in the present study is important because, over the recent years, there has been an increasing widespread use of relatively inexpensive "readymade" reading spectacles. This information can be used by manufacturers for horizontal lens centration; distance in such spectacles is intended for this Middle Eastern Arab population, and as such, this can avoid symptoms that may arise as a consequence of misalignment in the horizontal and vertical planes of the positive lenses before the wearer's eyes. ${ }^{22}$

\section{Limits of agreement in IPD obtained by same technique (within-technique) and across techniques (between techniques)}

IPD measurements obtained using the techniques employed in this study have been previously shown to have good repeatability in the same observer. ${ }^{2,9,18,21}$ However, Anderson ${ }^{2}$ claimed that repeated measures of FIPD repeatability was much better in the pupillometer-measured IPDs than in the Viktorin's method-measured IPDs. The current study did not explore repeatability and as such, verification of this claim was not possible.

The results presented here showed that the mean anatomical FIPD and NIPD obtained using the Viktorin's method was slightly smaller (although statistically insignificant) than the physiological IPD determined by PD-5 pupillometer by an average of $0.3 \mathrm{~mm}$ and $0.1 \mathrm{~mm}$, respectively. McMahon et $\mathrm{a}^{19}$ reported a similarly minimal but significant bias $(0.6 \mathrm{~mm})$ in FIPD, with the pupillometer returning larger IPDs. 
On the other hand, Holland and Siderov ${ }^{17}$ observed a nonstatistically significant difference $(0.08 \mathrm{~mm})$ in FIPD between both techniques when measurements were taken by the same examiner. However, when different observers measured FIPD on the same subject, the difference they observed was statistically significant.

In contrast, a study on Arab subjects reported that the anatomical IPD (determined by Viktorin's method) was on the average $0.10 \mathrm{~mm}$ and $0.30 \mathrm{~mm}$ wider than the physiological IPD (determined by PD-5 pupillometer, Essilor Ltd, Thornbury, Bristol, UK) from far and near, respectively. ${ }^{7}$ Even though the differences in anatomical and physiological IPDs found in the above cited study, and given that the observations in the present study were not statistically significant, the use of different pupillometers in the determination of IPDs has been reported to result in variations larger than $1.5 \mathrm{~mm} .{ }^{14}$ The discrepancy in pupillometer-measured IPDs is not caused by measurement uncertainties or setting errors made by the examiner, but is exclusively due to system-specific calibration differences and differing measuring principles. ${ }^{14}$

Also, between FIPD and NIPD, the differences were similar in both techniques $(4.4 \mathrm{~mm} \pm 1.1 \mathrm{~mm}$ and $4.6 \mathrm{~mm} \pm 1.0 \mathrm{~mm}, P>0.05$; Viktorin's and PD-5, respectively), and as such, both techniques can be interchangeably used in the clinical determination of male IPDs at both distances by same examiner. This is in agreement with the conclusion reached by a similar study carried out about 2 decades ago. ${ }^{18}$ Across all subjects, FIPD was consistently significantly larger than NIPD despite the technique used. The average bias was $4.4 \mathrm{~mm}$ (Viktorin's method) and $4.6 \mathrm{~mm}$ (PD-5); this is also similar to previous reports. ${ }^{6,7,10,12}$ Pointer $^{24}$ observed an intragender difference of a slightly lower magnitude (approximately $3 \mathrm{~mm}$ ) between far and near IPDs obtained by Viktorin's method in Caucasian presbyopes.

Grossvenor $^{37}$ calculated the differences between FIPD and NIPD obtained using a corneal reflection method ( $\mathrm{mm}$ rule) of alignment to be $3.5 \mathrm{~mm}, 4.0 \mathrm{~mm}$, and $4.5 \mathrm{~mm}$ for FIPDs of $55 \mathrm{~mm}, 63 \mathrm{~mm}$, and $71 \mathrm{~mm}$, respectively. The corresponding differences for the respective FIPDs of $3.9 \mathrm{~mm}, 4.4 \mathrm{~mm}$, and $5.0 \mathrm{~mm}$ (Viktorin's method); $4.0 \mathrm{~mm}, 4.6 \mathrm{~mm}$, and $5.2 \mathrm{~mm}$ (PD-5 method) observed in the current study were similar to that of Grossvenor. ${ }^{37}$ This is important in situations where the examiner is amblyopic in one eye. He/she will be able to measure the patients' NIPD using the normal eye and the appropriate amount based on these differences can be added to obtain the corresponding FIPD and/or the FIPD can be predicted from the linear equation expressed in the results above (Figure 3).

\section{Influence of age on interpupillary distance}

A statistically significant variation was evident in both FIPD and NIPD with advancing age, despite the technique used $(P=0.0001)$. The FIPD has increased by $2.8 \mathrm{~mm}$ and $1.8 \mathrm{~mm}$ (Viktorin's and PD-5, respectively) from the early adulthood years (16 to 25 years) to late adulthood (26-40 years). A decrease of $0.6 \mathrm{~mm}$ and $0.4 \mathrm{~mm}$ (Viktorin's and PD-5, respectively) occurred between the adults (26-40 years) and older adults (41-67 years). A similar but slightly different trend was shown in the NIPD (Table 2). Between early adulthood (16-25 years) and old age (41-67 years), the FIPD had increased by $2.2 \mathrm{~mm}$ and $1.3 \mathrm{~mm}$ (Viktorin's and PD-5, respectively), with the NIPD increasing by $2.8 \mathrm{~mm}$ and $2.3 \mathrm{~mm}$ (Viktorin's and PD-5, respectively). This indicates that in line with previous reports, ${ }^{6,9,38-41}$ the increase in FIPD and NIPD in early adulthood continues towards an individual's late $30 \mathrm{~s}$, and begins to decrease in the fourth decade of life.

Chen and $\mathrm{O}^{\prime}$ Leary ${ }^{8}$ revealed a significant difference in the IPD growth rate between men and women. Evereklioğlu et al ${ }^{10}$ postulated that the average total growth increment for NIPD and FIPD in males ( 7 and 35 years old) were $8.66 \mathrm{~mm}$ and $9.31 \mathrm{~mm}$, respectively. Fesharaki et al ${ }^{13}$ observed an increase in the mean FIPD of $4.8 \mathrm{~mm}$ during the second decade, $1.7 \mathrm{~mm}$ during the third decade, and $0.6 \mathrm{~mm}$ during the fourth and fifth decades of life.

This change in IPD with age can be explained from an anatomical view of the human orbit. ${ }^{9}$ It is recognized that in infants, the interorbital linear skeletal dimension is small relative to the globe size, which often gives the appearance of a pseudo-squint; ${ }^{9}$ this increases with the development of the nasal cavity, other sinuses, and the eruption of the teeth. The male continues to develop in the brow region during puberty, showing an increase in the width of the facial bones, which produces a horizontal-rectangular orbital entrance. This is quite different from female development during puberty; thus, the gender difference in the human facial skeleton. ${ }^{9}$ The fact that the bony orbit itself continues to grow into adulthood and only attains its full dimension at puberty, with subsequent changes occurring around the orbital margin in the elderly as a consequence of bone atrophy, explains the differences in the rate of IPD progression in the age groups reported in the current study. Therefore, the current findings confirm that age can cause bidirectional changes in IPDs, most likely due to the reported effects of menstruation changes that occur during childhood physical growth changes and other changes in 
body features (leading to a rapid increase in IPD) on the one hand, ${ }^{6}$ and the increased laxity of the soft tissues in the elderly subjects (leading to a decrease in IPD) on the other. ${ }^{39}$

\section{Influence of age and near interpupillary distance (NIPD) on near heterophoria}

Age has been strongly associated with stereopsis, fusional vergence, and the accommodative convergence/accommodation ratio. ${ }^{35,42}$ Normality of all of the above visual functions is responsible for comfortable single binocular vision, and has been reported to decrease with increasing age. A significant association has been shown to exist between age and phoria. ${ }^{8,34,35}$ In one study, ${ }^{8}$ about $98 \%$ of the children below 5 years of age were found to be predominantly exophoric at near. Anderson et $\mathrm{al}^{35}$ assessed different ocular functions including fusional vergences, near point of convergence (NPC), distance and near phoria, and IPD annually, over a period of 10 years in 114 subjects aged 7 to 13 years. They reported that the mean distance phoria at year 1 was 0.1 pd exophoria, and this did not change throughout the study period, whereas a significant $(P<0.001)$ shift in near phoria over the 10-year study period (2.4 pd esophoria to 4 pd exophoria) occurred. In the present study, heterophoria was positively associated with changes in age and NIPD (Figures 7 and 8, respectively) at a significant level. Subjects exhibited near esophoria up until their mid-30s, and beyond this stage, a shift towards exophoria was shown (Figure 7). A similar trend was also observed in the relationship between NIPD and the amount of near heterophoria (Figure 8). There was also a tendency for greater near esophoria in subjects with NIPD, which was smaller than $62.5 \mathrm{~mm}$, and greater near exophoria in subjects with larger NIPD. This indicates that, as noted by a previous study, ${ }^{8}$ there is a reduction in the ability of the oculomotor control system to converge to compensate for age-related changes in IPD. This oculomotor system is responsible for overcoming the increasing demand on total convergence as predicted from IPD during childhood; however, with age, this muscular capability decreases. Again, because the accommodative convergence or proximal convergence (which play a role in maintaining the process of orthophorization at early adulthood) also suffer from a depreciation in function due to aging, ${ }^{35}$ and with the observed decrease in near base-out ranges (compensating vergence for exophoria) from childhood to early adulthood not being accompanied by significant changes in near base-in ranges over this time period, ${ }^{43}$ we will therefore expect a predominance of exophoria (as observed in the current study) in the older age group.
On the other hand, the direction of the observed trend in this study may be logical given that individuals with a larger IPD will have a greater convergence demand for near tasks, and with increasing age, they will lose the ability to accommodate, thereby losing the benefit of accommodative convergence. Also, an increase in IPD with age affects the oculomotor function by increasing the amount needed for rotation of the eyes. ${ }^{8}$ When this happens, the demand on fusional vergence (base-out ranges) becomes greater, and a shift towards exophoria results, as was observed in the older adults. The probability for even greater exophoria, and a greater loss of compensating fusional vergence ability, results in near point symptoms, which is evident in the reading difficulty commonly experienced by the presbyopic subjects.

Accommodation and vergence help maintain single and focused visual experiences while an object moves in depth. Theoretically, the relationship between them requires constant recalibration as the head grows and hyperopia decreases. Jiang and Ramamirtham ${ }^{42}$ showed that the accommodative convergence/accommodation and convergence accommodation/convergence ratios of adults change when their IPD is artificially increased or decreased by wearing periscopic spectacles for 30 minutes. Again, the accommodative demand for a typical infant is larger than for an adult as a result of their hyperopic refractive error, ${ }^{44}$ while the vergence demand is smaller because of their narrow IPD. ${ }^{11}$ This effect is likened to the observation in the current study where the propensity to be esophoric at near was observed in subjects with smaller NIPDs, and a greater exophoria in subjects with larger NIPDs. Previous studies have observed similar age variations as in interocular distance, ${ }^{15}$ intercanthal distances, head circumference, ${ }^{12}$ axial length, and eyeball size and shape. ${ }^{45}$ Osuobeni and al-Gharni ${ }^{15}$ showed that interocular distance was wider in older subjects than in younger subjects, due to soft tissue changes resulting from aging. Osunwoke et $\mathrm{al}^{12}$ reported that intercanthal distances and head circumference were significantly smaller in both males and females of 3-7 years of age in relation to subjects aged 18-21 years. In another study, ${ }^{45}$ axial length, as well as eyeball size and shape were shown to increase with age. The study also observed that, in all subjects, the axial length increased rapidly from infancy until about age 10, after which no significant change occurred, whereas the change in eye shape (from oval to spherical or vice versa) was dependent on the refractive error of the subject.

Since all these parameters increase with age including NIPD, while accommodation depletes, ${ }^{42}$ it can then be deduced that, the farther away the eyes are from each other, 
the greater the demand on accommodation and fusional vergence (which are required to maintain single binocular vision). The tendency for the eyes to deviate outward (exophoria) as observed in the current study, may then not be unexpected.

While the current study has strength in the protocol of selection of our subjects; the visual functions hereby assessed; and the wider age range of subjects, which could enable generalization of the results in subjects of various age groups; it is also limited by the gender selection of the study. There is the need for further studies evaluating these ocular functions in both genders to assess the rate of change with increasing age.

In conclusion, the study offers population-specific data on anatomical and physiological FIPDs and NIPDs that can be used to aid in the development of spectacles, frames, and over-the-counter ready-made reading spectacles in this region to ensure a better fit and to ameliorate problems associated with misalignment. It is also useful in syndrome diagnosis. The study showed that: (1) FIPD and NIPD increases with age; (2) between FIPD and NIPD, differences as much as $6.6 \mathrm{~mm}$ and as low as $2.2 \mathrm{~mm}$ in Arab males should be expected; and (3) the mean anatomical FIPD and NIPD obtained using the Viktorin's method and the mean physiological IPD determined by PD-5 pupillometer in a clinic setting could be interchangeably used, insofar as both measurements are obtained by the same examiner. However, in such situations, caution should be exercised, especially in patients requiring high power refractive corrections as this difference could vary from $-4 \mathrm{~mm}$ to $+3 \mathrm{~mm}$ (FIPD) and $-3 \mathrm{~mm}$ to $+3 \mathrm{~mm}$ (NIPD). Such variations might be intolerable as a result of the induced prismatic effects. Also, NIPDs significantly influenced the phoria position of the eyes, as subjects exhibited greater near esophoria in smaller NIPDs, and greater near exophoria in larger NIPDs.

\section{Acknowledgments}

The authors extend their appreciation to the Research Centre, College of Applied Medical Sciences, and the Deanship of Scientific Research at King Saud University for funding this research.

\section{Disclosure}

The authors report no conflicts of interest in this work. No competing financial interests exist.

\section{References}

1. Quant JR, Woo GC. Normal values of eye position in the Chinese population of Hong Kong. Optom Vis Sci. 1992;69(2):152-158.
2. Anderson AL. Accurate clinical means of measuring intervisual axis distance. AMA Arch Ophthalmol. 1954;52(3):349-352.

3. Cesario VA Jr, Latta GH Jr. Relationship between the mesiodistal width of the maxillary central incisor and interpupillary distance. $J$ Prosthet Dent. 1984;52(5):641-643.

4. Juberg RC, Sholte FG, Touchstone WJ. Normal values for intercanthal distances of 5- to 11-year-old American blacks. Pediatrics. 1975;55(3):431-436.

5. Grobbelaar R, Douglas TS. Stereo image matching for facial feature measurement to aid in fetal alcohol syndrome screening. Med Eng Phys. 2007;29(4):459-464.

6. Osuobeni EP, Faden FK. Interpupillary distance of females of Arab origin. Optom Vis Sci. 1993;70(3):244-247.

7. Osuobeni EP, al-Musa KA. Gender differences in interpupillary distance among Arabs. Optom Vis Sci. 1993;70(12):1027-1030.

8. Chen AH, O'Leary DJ. Changes in the interpupillary distance with the age and its effect on the near fusion free position. Aust NZJ Ophthalmol. 1997;25(1):S6-S8.

9. Pointer JS. The far interpupillary distance. A gender-specific variation with advancing age. Ophthalmic Physiol Opt. 1999;19(4):317-326.

10. Evereklioğlu C, Doğanay S, Er H, Gündüz A. Distant and near interpupillary distance in 3448 male and female subjects: final results. Turgut Özal Tip Merkezi Dergisi. 1999;6(2):84-91.

11. MacLachlan C, Howland HC. Normal values and standard deviations for pupil diameter and interpupillary distance in subjects aged 1 month to 19 years. Ophthalmic Physiol Opt. 2002;22(3):175-182.

12. Osunwoke EA, Didia BC, Olotu EJ, Yerikema AH. A study on the normal values of inner canthal, outer canthal, interpupillary distance and head circumference of 3-21 years ijaws. American Journal of Scientific and Industrial Research. 2012;3(6):441-445.

13. Fesharaki H, Rezaei L, Farrahi F, Banihashem T, Jahanbkhshi A. Normal interpupillary distance values in an Iranian population. $J$ Ophthalmic Vis Res. 2012;7(3):231-234.

14. Wesemann W. Comparison of PD measuring devices. Part 2. Optician. 2010:22-28.

15. Osuobeni EP, al-Gharni SS. Ocular and facial anthropometry of young adult males of Arab origin. Optom Vis Sci. 1994;71(1):33-37.

16. Packiriswamy V, Kumar P, Rao KG. Photogrammetric analysis of palpebral fissure dimensions and its position in malaysian South Indian ethnic adults by gender. N Am J Med Sci. 2012;4(10): 458-462.

17. Holland BJ, Siderov J. Repeatability of measurements of interpupillary distance. Ophthalmic Physiol Opt. 1999;19(1):74-78.

18. Osuobeni EP, al-Fahdi M. Differences between anatomical and physiological interpupillary distance. J Am Optom Assoc. 1994;65(4): 265-271.

19. McMahon TT, Irving EL, Lee C. Accuracy and repeatability of self-measurement of interpupillary distance. Optom Vis Sci. 2012; 89(6):901-907.

20. Backman H. Interpupillary distance measurements. Am J Optom Arch Am Acad Optom. 1972;49(3):264-266.

21. Mattison-Shupnick M. Comparing methods and techniques for interocular distance measurements-part 1. Optometric Monthly. 1980;71:10-12.

22. Jonasson F, Thordarson K. Refraction and interpupillary distance measurements of adult Icelanders with special reference to presbyopes possible use of supermarket standard spectacles. Acta Ophthalmol Suppl. 1987;182:44-47.

23. Elliott DB, Green A. Many ready-made reading spectacles fail the required standards. Optom Vis Sci. 2012;89(4):E446-E451.

24. Pointer JS. The interpupillary distance in adult Caucasian subjects, with reference to 'readymade' reading spectacle centration. Ophthalmic Physiol Opt. 2012;32(4):324-331.

25. Sasieni LS. Principles and Practice of Optical Dispensing and Fitting, 3rd ed. London, UK: Butterworths; 1975:45-185.

26. Schor C, Ciuffreda K. Vergence Eye Movements: Basic and Clinical Aspects. London, UK: Butterworths; 1983:492. 
27. Goss DA, Patel J. Comparison of fixation disparity curve variables measured with the Sheedy Disparometer and the Wesson Fixation Disparity Card. Optom Vis Sci. 1995;72(8):580-588.

28. Corbett A, Maples WC. Test-retest reliability of the Saladin card. Optometry. 2004;75(10):629-639.

29. Frantz KA, Elston P, Michalik E, Templeman CD, Zoltoski RK. Comparison of fixation disparity measured by saladin card and disparometer. Optom Vis Sci. 2011;88(6):E733-E741.

30. Maples WC. Optometry's paradigm shift. Optometry. 2001;72(9): 567-569.

31. Zaba JN, Johnson RA, Reynolds WT. Vision examinations for all children entering public school - the new Kentucky law. Optometry. 2003;74(3):149-158.

32. Bland JM,Altman DG. Statistical methods for assessing agreement between two methods of clinical measurement. Lancet. 1986;1(8476):307-310.

33. Dodgson NA. Variation and extrema of human interpupillary distance. Woods AJ, Merritt JO, Benton SA, Bolas MJ, editors. Proceedings of the SPIE Stereoscopic Displays and Virtual Reality XI conference; January 19-22, 2004; San Jose, CA. 2004;5291:36-46.

34. Leone JF, Cornell E, Morgan IG, et al. Prevalence of heterophoria and associations with refractive error, heterotropia and ethnicity in Australian school children. Br J Ophthalmol. 2010;94(5):542-546.

35. Anderson H, Stuebing KK, Fern KD, Manny RE. Ten-year changes in fusional vergence, phoria, and nearpoint of convergence in myopic children. Optom Vis Sci. 2011;88(9):1060-1065.

36. Hofstetter HW. Normal values of interpupillary distance. $J \mathrm{Am}$ Optometric Assoc. 1972;43:1151-1155.
37. Grosvenor T. Primary Care Optometry 5th ed. St. Louis: Butterworth; 2007. pp.183-208.

38. Pryor HB. Objective measurements of interpupillary distance. Pediatrics. 1969;44(6):973-977.

39. Fledelius HC, Stubgaard M. Changes in eye position during growth and adult life as based on exophthalmometry, interpupillary distance, and orbital distance measurements. Acta Ophthalmol (Copenh). 1986;64(5):481-486.

40. Brückner R, Batschelet E, Hugenschmidt F. Basal longitudinal study on aging (1955-1978). Ophthalmo-gerontological research results. Doc Ophthalmol. 1987;64(3):235-310.

41. Lakshminarayana P, Janardhan K, David HS. Anthropometry for syndromology. Indian J Pediatr. 1991;58(2):253-258.

42. Jiang BC, Ramamirtham R. The adaptive effect of narrowing the interocular separation on the AC/A ratio. Vision Res. 2005;45(20): 2704-2709.

43. Scheiman M, Wick B. Primary care of binocular vision, accommodative, and eye movement disorders. In: Clinical Management of Binocular Vision: Heterophoric, Accommodative, and Eye Movement Disorders, 2nd ed. Philadelphia, PA: Lippincott Williams \& Wilkins; 2002: $118-121$.

44. Aslin RN, Jackson RW. Accommodative-convergence in young infants: development of a synergistic sensory-motor system. Can J Psychol. 1979;33(4):222-231.

45. Song HT, Kim YJ, Lee SJ, Moon YS. Relations between age, weight, refractive error and eye shape by computerized tomography in children. Korean J Ophthalmol. 2007;21(3):163-168.
Clinical Ophthalmology

\section{Publish your work in this journal}

Clinical Ophthalmology is an international, peer-reviewed journal covering all subspecialties within ophthalmology. Key topics include: Optometry; Visual science; Pharmacology and drug therapy in eye diseases; Basic Sciences; Primary and Secondary eye care; Patient Safety and Quality of Care Improvements. This journal is indexed on

\section{Dovepress}

PubMed Central and CAS, and is the official journal of The Society of Clinical Ophthalmology (SCO). The manuscript management system is completely online and includes a very quick and fair peer-review system, which is all easy to use. Visit http://www.dovepress.com/ testimonials.php to read real quotes from published authors. 